drs. Hubertus van Rijt

\title{
„Wir bringen ältere und jüngere Kollegen zusammen“
}

\begin{abstract}
Praxisabgabe und Praxisübernahme - dieses Thema ist zu einem großen Feld der Zukunftsaufgaben des FVDZ geworden. Bundesvorstandsmitglied Hubertus van Rijt hat sich in den vergangenen zwei Jahren engagiert für eine Neustrukturierung des Bereichs eingesetzt und viele Ideen entwickelt, um ältere Kollegen und niederlassungswillige Zahnärzte zusammenzubringen.
\end{abstract}

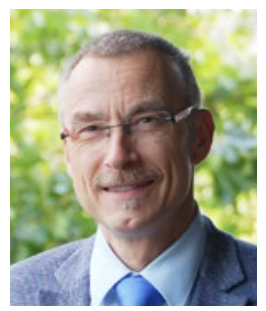

\section{Wo stand der FVDZ, als Sie den Bereich Praxisübergabe übernommen haben?}

Vor zwei Jahren gab es beim FVDZ eine Online-Stellenbörse. Diese Börse war allerdings eher unstrukturiert. Es gab ein munteres Durcheinander von Stellengesuchen und -angeboten für angestellte Zahnärzte, eine Suchfunktion nach Regionen oder Spezialisierungen war allerdings nicht vorhanden. Das Thema Praxisabgabe oder die Suche nach einer Praxis zur Übernahme war nur begrenzt möglich. Deshalb wurde der FVDZ nicht als kompetenter Partner für die Vermittlung wahrgenommen.

\section{Was haben Sie in den zurückliegenden zwei Jahren erreicht?}

Wir haben zunächst einmal die Problemlage analysiert: Was können wir als Verband für unsere Kollegen tun, die nach langer und erfolgreicher Tätigkeit ihre Praxis abgeben wollen. Denn die älteren Zahnärzte stehen ganz klar vor dem größeren Problem: Es gibt mehr Praxen, für die ein Nachfolger gesucht wird als jüngere Zahnärzte, die sich niederlassen wollen. Der FVDZ hat sich intensiv in seinem Mentoringprogramm auch mit diesem Thema auseinandergesetzt. Allein mit einem Online-Stellen- oder Praxismarkt ist es nicht getan, sondern es kommt sehr auf den persönlichen Kontakt an. Beim Mentoringtreffen in Amsterdam und Haarlem in diesem Frühjahr habe ich von den Nöten der jungen Zahnärzte gehört. Es wurde dann sehr deutlich, dass es gerade auch für die jungen Kollegen großen Bedarf an Aufklärung und Austausch gibt. Wir haben erstmals die beiden Gruppen direkt zusammengebracht.

Derzeit arbeiten wir daran, die Praxisabgabe und -übernahme zu strukturieren, so dass bereits in der Online-Suchphase eine Vorsortierung stattfinden kann. Wir haben gesehen, dass sowohl diejenigen, die eine Praxis suchen, sehr genaue Vorstellungen und Wünsche haben als auch diejenigen, die eine Praxis abgeben. $\mathrm{Zu}$ bieten haben beide Seiten etwas - wir wollen sie zusammenbrin- gen. Für den Verband ist das eine große und schöne Aufgabe, weil sie junge und ältere Mitglieder einbezieht. Auch an der Neustrukturierung für Stellengesuche und Stellenangebote für angestellte Zahnärzte, Vorbereitungsassistenten oder Praxisvertretungen wird weiter gearbeitet. Die Vermittlung von Partnerschaften möchten wir auch in das Konzept mit aufnehmen.

Wo wir jetzt stehen? Wir arbeiten daran, den FVDZ in diesem Bereich zu modernisieren, so dass er für die jüngeren Mitglieder, selbstständig oder angestellt tätig, aber trotzdem freiberuflich, ebensoviel bietet wie für die älteren. Das Mentoringprogramm ist ein großer Baustein dafür. Und ganz nebenbei haben wir im Praxishandbuch die Checklisten und Empfehlungen zur Praxisabgabe überarbeitet, so dass jetzt alles aus einem Guss ist.

\section{Was ist das Ziel für die nächste Wahlperiode?}

Wir werden die Ideen, die wir jetzt für die Vernetzung von Praxisabgebern und Praxissuchenden entwickelt haben, in die Tat umsetzen. Wichtig ist es, dass wir die jungen Zahnärzte früh abholen - als Studenten oder angestellte Zahnärzte - und die älteren Kollegen mitnehmen. Zunächst einmal wollen wir eine Art „Online-Praxispool“ einrichten. Dort kann man sich über die Bundesgeschäftsstelle anmelden. Nach Überprüfung der Daten wird das, was derjenige sucht oder anbietet, regionalisiert und detailliert eingestellt. Aber anders als auf einer DatingPlattform im Internet soll es einen echten Menschen geben, der die Anbieter und die Suchenden mit ihren Ansprüchen und Anforderungen vorsortiert und miteinander in Kontakt bringt. Eine Art Praxis-Headhunting, wenn man so will. Das wird sehr viel Arbeit für die Suchenden auf beiden Seiten ersparen. Von heute auf morgen wird das aber nicht zu stemmen sein. Gleichzeitig wollen wir die Mentoringtreffen zur Praxisabgabe und Praxisübernahme regional weiterführen. Daran besteht nach dem ersten Treffen ein großer Bedarf.

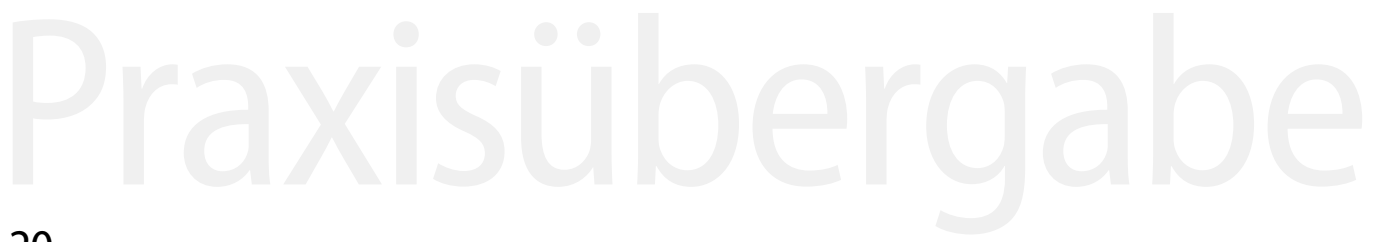

